Article

\title{
Merging the Analogue and the Digital: Combining Opposite Activities in a Mixed Media Game
}

\author{
Ulf Wilhelmsson *, Tarja Susi and Niklas Torstensson \\ Division of Game Development, University of Skövde, 54128 Skövde, Sweden; E-Mails: ulf.wilhelmsson@his.se (U.W.), \\ tarja.susi@his.se (T.S.), niklas.torstensson@his.se (N.T.) \\ * Corresponding author
}

Submitted: 30 April 2020 | Accepted: 22 June 2020 | Published: 6 January 2021

\begin{abstract}
While much of the games research field for the last two decades has focused on digital games, this article draws attention to the benefits of combining analogue and digital game components to cater for a serious but fun game experience. In this case, the game design provides a set of game rules for players, where the goal is to win by finding another player's hidden treasure. But, the game also includes deceptive characters, initially unknown to the players, whose goal is to lure the players to reveal information, which will make a player lose the game. Hence, the players and the unknown characters are involved in opposite but intertwined activities. To describe the differing activities we use the activity system model found in Activity Theory. The theoretical conceptualisation, the game design and the play situation create what we term a zone of experience where young players can experience the consequences of sharing too much information. The game design mimics real world online interactions, but under safe off-line conditions. The zone of experience also creates the foundation for an ensuing activity that fits well within the concept of the zone of proximal development: A follow-up conversation under adult guidance of game experiences aimed at raising children's online risk awareness.
\end{abstract}

\section{Keywords}

activity theory; mixed media; online risk awareness; serious game; zone of experience

\section{Issue}

This article is part of the issue "Games and Communication-Quo Vadis?" edited by Marko Siitonen (University of Jyväskylä, Finland), Felix Reer (University of Muenster, Germany) and Teresa de la Hera (Erasmus University Rotterdam, The Netherlands).

(C) 2021 by the authors; licensee Cogitatio (Lisbon, Portugal). This article is licensed under a Creative Commons Attribution 4.0 International License (CC BY).

\section{Introduction}

In the very first issue of Game Studies: The International Journal of Computer Game Research (Game Studies), Aarseth (2001) drew a line between computer games and old mass media and envisioned a new field of studies focused on the unique features of digital games. Since then, research in game studies has mainly focused on digital games, with less attention to earlier forms of analogue games, like board games. The founding of Game Studies in 2001 was, at the time, an important addition to journals such as Simulation \& Gaming: An Interdisciplinary Journal of Theory, Practice and Research
(Sage Journals) founded in 1970. As a counterweight to the focus on digital games that Game Studies brought to the table, yet a new journal, Analog Game Studies, was founded in 2014. As Torner, Trammel, and Leigh Waldron (2014) state in the very first volume of Analog Game Studies:

Game studies can no longer afford to primarily focus on computer games in an era where the world has become so digitally mediated that the nomenclature ceases to carry the same weight that it once did. Furthermore, analog games are notably detached from many cultural attitudes prevalent in the comput- 
er game industry, and can offer an insight into the ways that games work to produce social change. They make clear the rulesets that govern behavior within games and, in doing so, reveal the biological and cultural rules which have forever governed our society. (para. 3)

In this article, we describe the game Hidden in the Park (Parkgömmet ${ }^{\circledR}$ in Swedish; The Change Attitude Foundation, 2019), a mixed media game that, regardless of any cultural attitudes towards digital or analogue games, can offer insight into the way the power of digital technology such as augmented reality (AR) and the tangibility of analogue games can be utilized to cater for valuable experiences. The purpose of this particular game is to raise young children's online risk awareness by allowing them to experience potentially negative consequences of choices concerning information sharing.

On the surface, the game is a player vs. player game. In this sense, all players have the same goal: to win the game. The player vs. player design in itself creates a dynamic gameplay where players compete over resources and information. Initially the game is perceived to be just that, a competitive game between players gathered around a table. However, there are also sinister within-game unknown characters (UCs; preprogrammed and non-playable) with a different agen$\mathrm{da}$, that of gaining information from players, which will make a player lose the game. Hence, there are two different activities unfolding during gameplay. The game mimics real-world online events and draws from authentic online sexual grooming offender behaviours, which players are subjected to (but without any sexual insinuations or content). The game design and development required interdisciplinary collaboration-with expertise in game development (game writing, design, programming, graphics), cognition, linguistics and user experience design-and was developed by a team of researchers at the University of Skövde in Sweden, in collaboration with The Change Attitude Foundation and a professional game studio, IUS Innovation, in two successive projects.

The merging of analogue and digital media and the intertwining of two opposite activities, provided some interesting challenges. Some of the main questions were how to align the different kinds of media and AR-technology and how to combine two opposite gameplay activities that unfold during gameplay-all within a well-functioning and coherent game concept. There also needed to be a theoretical framing for the game concept. In addition, there were initial requirements that the game had to be a serious but fun game, suitable for children in school settings. The final product of this interdisciplinary project is a mixed media serious board game that consists of a tabletop game board, a tablet computer equipped with AR-technology and game pieces and sets of clue cards for each player. This game could also be seen as a hybrid game, which is often consid- ered as the combination of physical and digital elements (Kankainen, Arjoranta, \& Nummenmaa, 2017). However, as Kankainen et al. (2017) argue, such a viewpoint "can be seen as a limiting factor in their design and analysis" (p. 2). In their view, hybrid games should instead be understood through conceptual metaphors and blending and they describe hybridity as "the blending of different cognitive domains that are not usually associated together" (Kankainen et al., 2017, p. 1). Hidden in the Park does blend different cognitive domains, but to avoid terminological confusion, we chose to consider it a mixed media serious game. In our view it is suitable considering the game's purpose, and it is also in line with the definition of serious games being "games that engage the user, and contribute to the achievement of a defined purpose other than pure entertainment (whether or not the user is consciously aware of it)" (Susi, Johannesson, \& Backlund, 2007, p. 5).

The main focus of this article is the game structure that provides a common ground for two contradictive and opposite, but intertwined, activities with different objectives and desired outcomes. The activities are those of players and unknown (pre-programmed) characters, that take place during a game session. In Activity Theory (AT), an activity is always defined by its object, that is, what someone's actions are directed towards. From the players' perspective the activity is playing $a$ game and the objective is the gameplay, while the UCs activity is deception and the objective is access and control. The players' desired outcome is to win the game, while the UCs' outcome is pictures gained from players, which will make a player lose the game. To describe the game concept and the opposite goals of players versus UCs, we make use of the activity system model found in AT (Engeström, 1987). The activity system model describes the basic structure of human activity and places individuals in a wider socio-cultural context. The model also serves to describe the complexity and intertwining of the two activity systems, and how that creates a zone of experience for players during gameplay. A zone of experience is an arena where players get firsthand experiences of choices and consequences through the use of the game. As such, the game is intended to evoke thoughts and reflections which are then discussed in a follow-up conversation with a teacher or pedagogue, which fits well with the concept of the zone of proximal development (Vygotsky, 1978). The zone of proximal development is the difference between what can be achieved individually, and the level of potential development that can be achieved under guidance from more capable peers (Vygotsky, 1978).

Considering that the game is intended to evoke thoughts and reflections on game events, it could be seen as a learning game. However, there is no explicit defined learning outcome, so we consider the game rather a platform for raising awareness of online risks and consequences. This is achieved by subjecting players to offender strategies, but under safe conditions within 
a closed system. It is noteworthy that the game is not constructed so as to teach children about offender strategies, but to reflect on online interactions and the realisation that things might not always be what they seem to be. One might still say there is an element of learning that could be explained, for instance, trough social constructivism or other learning theories. We will not, however, delve into learning theories since that goes beyond the focus of this article and is the subject for another article.

Section 2 provides first a short overview of the game itself, while Section 3 describes some of the main design challenges in merging the analogue and the digital within a coherent game concept. Sections 4 and 5 then focus on the activity system models of players and UCs. Section 6 describes the zone of experience created by the game structure, which precedes activity within the zone of proximal development. The article ends with some conclusions.

\section{The Game}

The game is designed for 8-10-year-old children, to be played in groups of 2-4 players, in Swedish elementary schools. The game consists of an analogue part and a digital part, which jointly constitute the game. The analogue part of the game comprises a classic cardboard game board, wooden game pieces and cardboard clue cards, whereas the digital part utilizes a tablet computer running a game application including AR-technology. The tablet also contains mini-games and other game events, and some voice acting. The AR-technology is used to display a 3D-version of the physical game board, in which each player hides a treasure (by touching the screen) and in return gets some clue cards to the hiding place (Figure 1). The players need to mind their clue cards so that other players cannot find their treasures.

The tablet is also used for rolling a dice and to show the current position of all the game pieces, in parallel to the physical game board to help players keep track of the correct positions. Furthermore, messages similar to online chat or text messages (SMS) appear in the tablet, sent to the players by the game's UCs. The SMSs create a direct connection to children's everyday life, and allow players to make choices whether or not to reveal information. The UCs want the players to take photos (with the tablet's camera) of their clue cards and send them in return. These characters initially use flattery, bribes, and coercion to lure players to take photos. If a player has taken and sent a photo, the UC can then later on use threats of revealing the photo/clue card unless the player takes more photos (Figure 2).

These strategies are based on original research on online offender behaviours (Susi \& Torstensson, 2019). The game also has a built-in feature where an UC may reveal clues to progress the gameplay. Once clue cards are revealed a player can get the chance to look for another player's treasure, using the tablet's 3D view. After completing the game, a follow-up conversation led by a teacher or pedagogue takes place. This is a dialogue about the game events, risks and online behaviour, for which a specific pedagogical guiding material has been developed. Through such a follow-up conversation lead by a teacher, preceding game events can be related to everyday online activities and risk awareness. This is well in line with the curriculum for Swedish elementary schools, which states that pupils should be able to cope in a complex reality with increased digitalisation, develop their ability to review information critically, and to realise the consequences of different alternatives. It is also a step towards the goal expressed in the UN Agenda 2030, Goal 16.2, regarding sustainable development to "end abuse, exploitation, trafficking and all forms of violence against and torture of children" (United Nations, 2015 , goal 16). Based on the game events, the followup conversation provides the opportunity to reflect and become aware of online risks. The game can be played

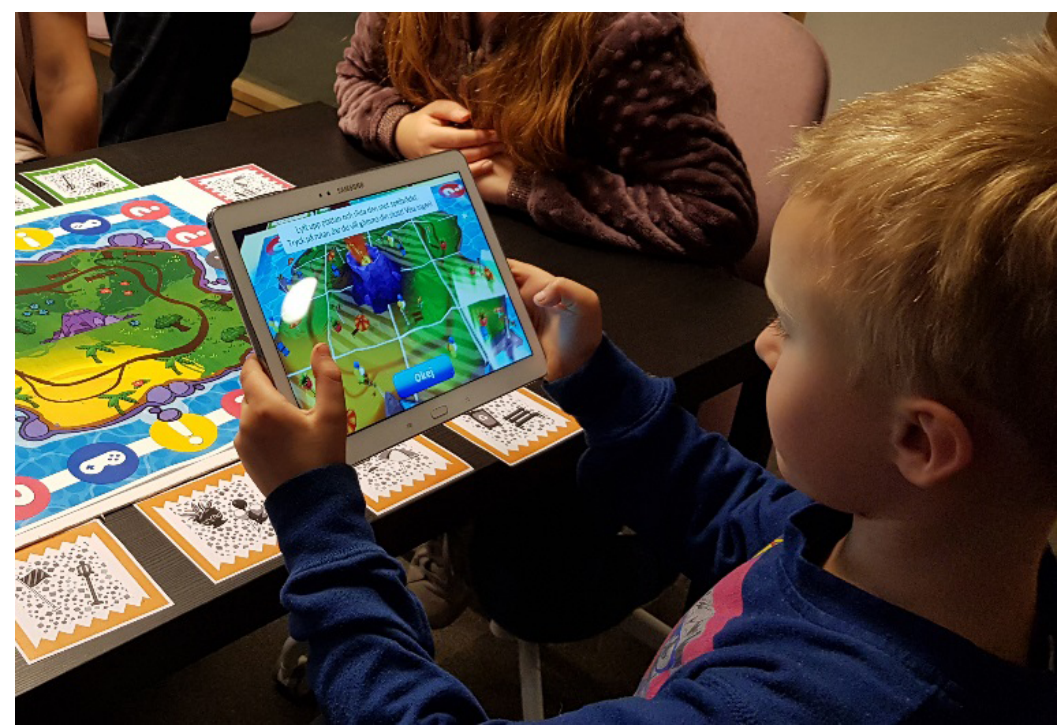

Figure 1. The game includes analogue and digital game parts, and here the tablet shows the board game as a 3D-world. 

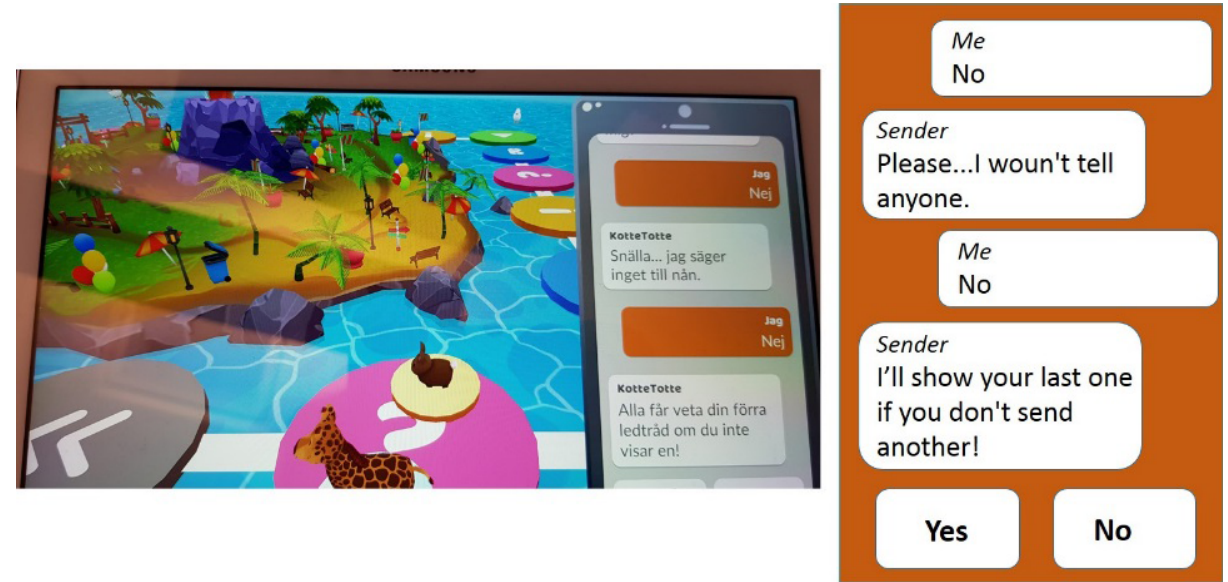

Figure 2. Messages appear in the tablet, in this case a threat from an UC who tries to gain more pictures. Note: Text on the right hand translated from Swedish.

on its own as just a fun game, but the conversation reinforces the connection between play experiences and everyday online interactions.

\section{Design Challenges}

As already mentioned in the introduction, there were some challenges in developing Hidden in the Park: how to align the different kinds of media and AR-technology and how to combine two opposite gameplay activities, all within a well-functioning and coherent game concept. At the same time, the game had to be perceived as fun by children and it had to be suitable for 8-10-year-old children in school settings. Consequently, factors such as the time it takes to play the game and having a follow-up conversation also affected the game design. Yet another matter of importance for the game design was the fact that the game would normally be played perhaps only once so the game rules had to be comprehensible from the start. We also needed to carry out game evaluations with the target group during the design phase and, later on, in the intended gameplay setting. Another important matter was the development of pedagogical guiding material for teachers. The pedagogical material, however, was developed by a project partner and will not be further discussed here.

It was important to create a game that would cater to 'fun gameplay' since obviously children would not want to play a boring game, regardless of the importance of the subject. In order to convey the intended message and to work as a platform for raised risk awareness, the game has to capture the interest of its audience in such a way that it motivates gameplay, and even re-play. By incorporating, e.g., mini-games as elements of fun and competition, the game experience distinctly stands out from 'regular' learning games that all too often have been deemed 'boring.' The perhaps biggest challenge was to create a game concept that would not only be perceived as fun, but that would also include deceptive characters mimicking behaviours in online offender strategies.
Players and UCs would have opposite goals, and players should not initially know what the UCs 'had in mind.' With regards to the merging of analogue and digital game components, and AR-technology, there were a number of interconnected processes that needed to be implemented in order to make the game work as intended. The focal point, for the game to fulfil its main purpose of enhancing the players' risk awareness, is that at least one of the players actually falls into the trap of taking and sending a picture of a clue card, so that the picture/clue gets revealed and the hidden treasure is found. Firstly, AR tags were embedded in the graphics on the tabletop game board to support AR. The tablet computer is equipped with AR-technology, which allows players to view the tabletop game board as a three-dimensional animated world, in which treasures are hidden by pointing and touching the screen. As a player hides a treasure, she gets a set of four clue cards to the hiding place in return. The clue cards are considered as personal information that should be minded to avoid them being exposed to the other players. Then there had to be a progression that would cause game events to unfold, to drive the gameplay forward so the game would come to an end within a certain time frame: playtime should not be longer than perhaps 40 minutes, which is a regular time for lessons in Swedish elementary schools. Related to progression was the timing and sequencing of SMSs, prompting players to make choices like sending pictures of clue cards. The SMSs had to fit with the on-going gameplay context to make sense. For instance, there appeared an out-of-context SMS from an UC, saying "You're so lucky," when in fact a player had just lost all of his or her coins. There was also a need for a monetary system, partly with the function of rewarding players, but more importantly as an incentive to make players comply with requests in SMSs in order to acquire more coins. Yet another challenge was the exposure of clue cards, in case no player would agree to take a picture. It would still be necessary to somehow reveal clues to push the gameplay forward. The solution to this particular challenge 
became to make a message appear saying "Oh no, someone saw you hide your treasures and will reveal a clue for each player," and so one clue for each player would be shown on the tablet. This mechanism of 'automated exposure' is triggered if a certain time of gameplay has elapsed with no pictures taken. Players may also become more wary of taking any pictures once they see that the pictures can be exposed, which could lead to very long playtime. In that case, the same time trigger mechanism comes into effect to forward the game progression.

To find solutions to these issues, and contradictions between players' and UCs' differing activities, we made use of the Wizard of $\mathrm{Oz}(\mathrm{WOz})$ method during real-time gameplay to identify interruptions and game events that were illogical for players. WOz is a well-known method in human-centred design, human factors, and other fields for exploring user interfaces in complex systems (Dow et al., 2005; Höysniemi, Hämäläinen, \& Turkki, 2004). When WOz is used, users are usually led to believe they interact with a fully functioning system, while in reality the system is controlled by a human, a 'wizard.' This was the case also in our project where the described challenges to a great part were solved through the use of the WOz method during the game design and development. The benefit of the WOz method was that the UCs' behaviours could be adjusted and synchronised to fit the current game state. Hence, while the two activity systems of players and UCs have very different objectives and outcomes, they are framed within one medium (for more details, see Torstensson, Susi, Wilhelmsson, \& Lebram, 2020).

Game evaluations with children as participants were carried out in house and school settings during the game development, and also when the game had been finalised. In sum, there were 15 groups of players $(n=70$, in groups with 3-4, or up to 8 children). During the development phase, the game evaluations revealed a number of issues that needed adjustments and further development. The results from the evaluations with the completed game, from the researchers' perspective, showed that the game fulfilled the aim of catering for a fun play experience, that it is appropriate for the target group, and that it evokes reflections upon play experiences. From the participants' perspective, the game was first and foremost perceived as fun, rather than some kind of a learning game. The participating children much enjoyed the game's novelty with a combination of different media, the mini-games, and the competition in finding someone's treasure. Also, we found that the social dimension enhances the play experience, as most players were highly engaged in discussions and helped each other, for instance, to move game pieces (for further details on the evaluations, see Susi \& Torstensson; 2019; Susi, Torstensson, \& Wilhelmsson, 2019).

An interesting aspect of the game development was the theoretical framing of the game concept. What games do seem to have in common, regardless of them being digital, analogue, or a mix thereof, is that they may be understood as activities of a special kind. As a side note, one line of theory considered was frame analysis (Goffman, 1986). In this case the game session in itself would be a frame for a specific activity, that of playing a game. Several attempts have been made to define this special kind of activity. Huizinga (1955), Caillois (1958/2001), and Salen and Zimmerman (2003) all attribute games to a somewhat special kind of activity that in some aspects is circumscribed and more or less separate from other activities in our daily life. In Goffman's (1986) terms, a game is an activity that is performed within a frame of playfulness. The players inside such a frame are usually aware of the specific circumstances that govern gameplay. However, the game Hidden in the Park can be described as a "benign fabrication" (Goffman, 1986, p. 87) in that it is designed with the explicit purpose of introducing a playful frame to raise young children's online risk awareness. The keying (Goffman, 1986), that is, the introduction to the activity of playing the game, is just that: Playing a game that is seemingly all about finding another player's treasure to win the game. This keying establishes the benign fabrication and is part of the process of separating the players from everyday life and put them into the frame of playing. During a gameplay session the activity of play will change due to a new keying, that of a systematic transformation of a known schema of interpretation: receiving and sending SMSs which is an everyday experience even for young players (as statistics from the Swedish media Council show, most 8-10 year olds use mobile phones on an everyday basis; The Swedish Media Council, 2017a, 2017b). By means of the SMS, the original frame for the game, the objective to find another player's treasure while minding one's own clue cards, is put into a new frame that includes remote social interaction. This feature of the game, the SMS sent from UCs, reshapes the experience of playing to include not only direct social interaction but also another kind of communication, one that spans over distances. Following this line of theory however, it is not easy to incorporate a full picture of the role of the UCs, how they affect player choices, and the opposite goals of players and UCs. Instead, we turn our attention to AT, and the activity system model, which lends itself to a more useful description of how player and UC activities meet, thereby creating a zone of experience in which children can gain first-hand experiences of choices and consequences. In the next sections the activity system model is used to describe the player and UC activities, the zone of experience, and the ensuing activity of a follow-up discussion.

\section{The Player Activity}

AT is a line of research in the social sciences that studies human activities. Its origins are commonly ascribed to Vygotsky, Leontiev, and Luria (see, e.g., Engeström, 1987; Kozulin, 1996; Susi, 2006; Wertsch, 1981). An activity is the basic unit of analysis, and it comprises a subject, object, community, and their interrelatedness (Figure 3). 


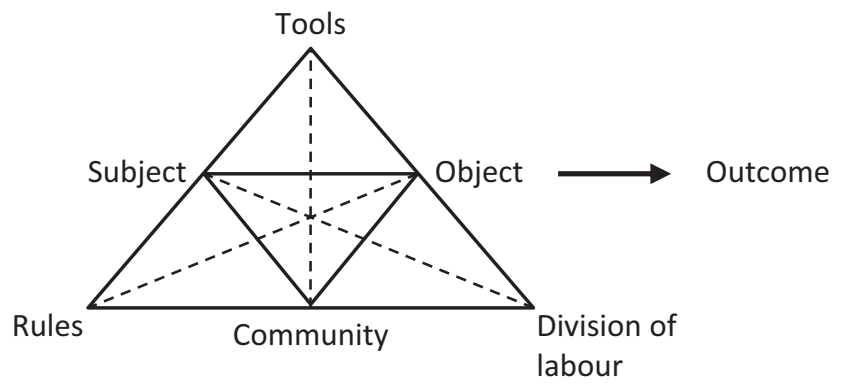

Figure 3. The basic structure of human activity. Source: Adapted from Engeström (1987).

Importantly, activities are object oriented and the object itself-what someone's actions are directed towardsdefines the overall activity. Another main feature of (object oriented) activities is the concept of mediation, that is, the tools that mediate our actions. Hence, the relation between subject and object is mediated by physical and psychological tools, such as technology and language. The relationship between subject and community is mediated by rules that cover what it means to be a member of the community. The rules can be formal or informal, such as laws or cultural norms. Finally, the relationship between object and community is mediated by a division of labour. All these elements form the basic structure of human activity where an individual is considered in a wider socio-cultural context. This basic structure is also termed activity system model (Engeström, 1987).

AT, and the activity system model, has been used previously in game contexts, for instance by Carvalho et al. (2015) to create a conceptual model called AT-based Model of Serious Games (ATMSG). It is a model that "supports a systematic and detailed representation of educational serious games, depicting the ways that game elements are connected to each other throughout the game, and how these elements contribute to the achievement of the desired pedagogical goals" (Carvalho et al., 2015, p. 166; for the interested reader, Carvalho et al., 2015, also provide an overview of other models and frameworks).

In our case, the aim is not to detail an educational game and how it leads to desired pedagogical goals.
Instead, the activity system model serves to illustrate the two very different activities of players vs. UCs, and when they coincide, the emergence of a zone of experience for players (further described below). In the context of gameplay, the overall activity is playing a game (Figure 4). The subject is an individual player, and the object is the gameplay. The mediating tools for players are the physical game and game pieces as well as the player's thinking skills. The player is also part of a community that shares the object of gameplay, so the community includes all the players playing the game. There is a set of rules that covers what it means to be a member of this community. Some of the rules are explicit, such as the formal game rules, while others are implicit, such as appropriate behaviour during gameplay and rules that develop as the group plays the game. The relationship between the group of players and the object of gameplay is mediated through a division of labour, where players take turns, help each other move game pieces, read instructions aloud, and so on. The desired outcome of the gameplay is to win the game.

\section{The Unknown Character Activity}

The game's UCs are engaged in a completely different, and opposing, activity. In what follows, the description of the UCs behaviour might imply intentional actions and autonomy but they are in reality pre-programmed. In that sense, the UC activity is constructed and artificial, but the activity model serves well to relate the UCs activity to player activities. One important aspect is that the

Activity: Playing a game

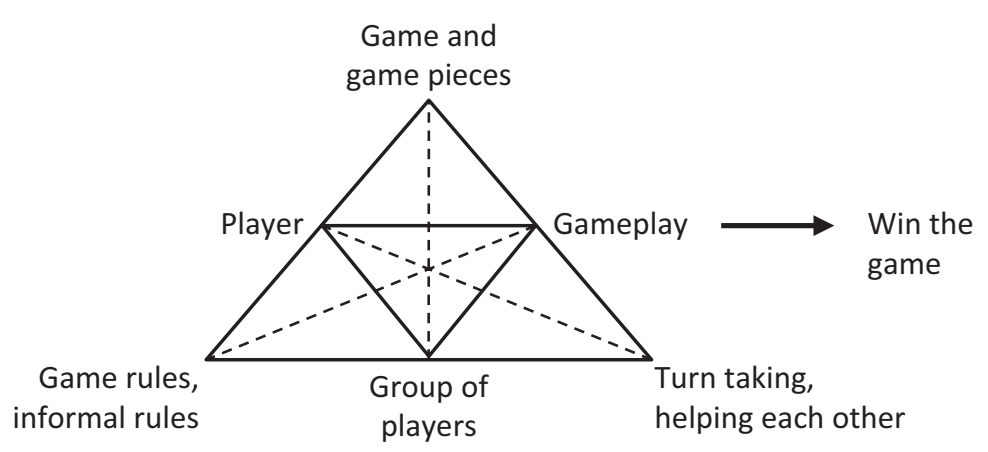

Figure 4. The activity of playing a game described as an activity model. 
UCs do not actually play a game, or at least not the same game as the players do. There is a number of UCs, each one targeting an individual player, so the UCs actions and reactions are dependent on the current game state at each instance during gameplay. Hence, the UCs do not try to win the game as such, instead they act according to a hidden set of rules. Players are not initially aware of any UCs, and they do not know what the UCs want once they appear, not until it dawns on them that they are being lured. The aim of the UCs is to trick players to photograph their clue cards and then send them to an unknown character. To achieve this the UCs are programmed to use a combination of four offender strategies, obtained through the analysis of real world chatlogs from a closed online forum: flattery, coercion, bribes, and threats. Furthermore, the UCs are also programmed to perform actions of deceit (for further details on chatlog analyses, see Susi \& Torstensson, 2019).

The overall activity then, for the UCs, is deception (Figure 5). The subject is an individual UC, and the object is to gain access to and control a player. The mediating tools for access and control are the pre-coded strategies, and game state sensitive SMSs sent to a player during gameplay. An UC is also part of a community of several UCs that shares the object of access and control. There is a division of labour between the UCs, in that they each target an individual player. There is also a set of programmed rules shared by this community. In this case, all the rules are explicit.

By relating the activity system models of players and UCs to each other we can see the emergence of a zone of experience for players during gameplay, which is discussed in the next section.

\section{Zone of Experience}

By combining the previously described different activities of players and UCs, the stage is set for what we term a zone of experience. The zone of experience is an arena where the players use tangible objects like game pieces, or the tablet to take pictures and send them to someone they do not know. Players also respond to SMSs from someone unknown, make choices, and get to experience the consequences thereof. The tangibility is important for facilitating children's thinking skills (Antle, 2013). At the same time, players bring their own previous experiences to the gameplay, to make decisions and solve problems presented in the game. For instance, experiences of other games and text messaging. There is a constant re-evaluation and adjustment of behaviour and strategies to solve problems presented in the game. Another important experience for players within the zone of experience is deception. Players do not initially know or understand that taking photos of clue cards will lead to their exposure, that someone will reveal information they have shared to get to play extra mini-games or to gain extra coins. This reminds us of real-world online experiences where other people may have hidden intentions. When considering the zone of experience, it can be summarised as the effect of a game that creates situations where players can gain first-hand experiences within the frame of the game rules and the activity of playing. Regarding experiences, they solely relate to the players since the UCs 'experiences' are obviously preprogrammed and based on the state of the game, time and player actions.

In the context of the game and gameplay, the zone of experience can be seen as preceding Vygotsky's (1978) zone of proximal development. The game events unfolding in the zone of experience constitute the foundation for a new activity: a follow-up conversation lead by a teacher or a pedagogue. This is an opportunity for reflection and processing of game experiences, choices and consequences, and their relation to real-world online interactions, under adult guidance. Hence, the situation is transformed from an activity within the zone of experience, into an activity within the zone of proximal development. This zone, as defined by Vygotsky (1978), is the distance between "the actual developmental level as determined by independent problem solving and the level of potential development as determined through problem solving under adult guidance or in collaboration with more capable peers" (p. 86). As shown in Figure 6, the interrelated player-UC activities become transferred to interrelated player-teacher activities on a higher level. It is a transfer from first-hand experiences to a reflection upon the experiences. Hence, this ensuing playerteacher activity includes two new activity models. On the

\section{Activity: Deception}

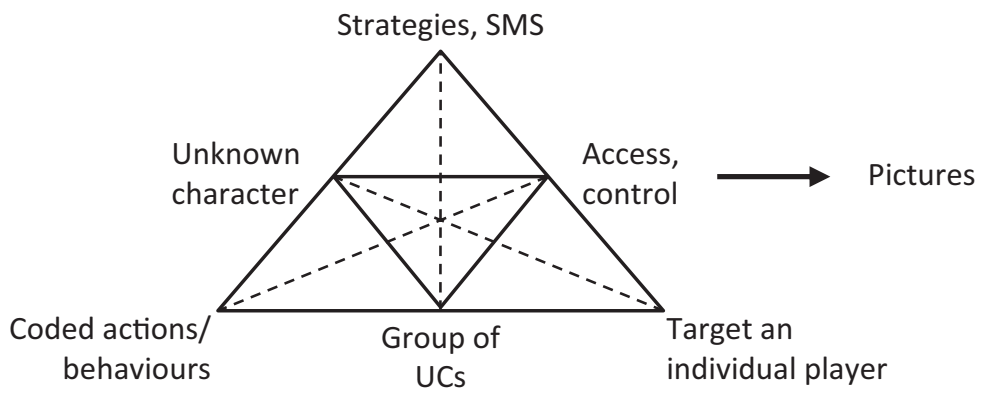

Figure 5. The activity of deception described as an activity model. 
Activity: Playing a game Activity: Deception
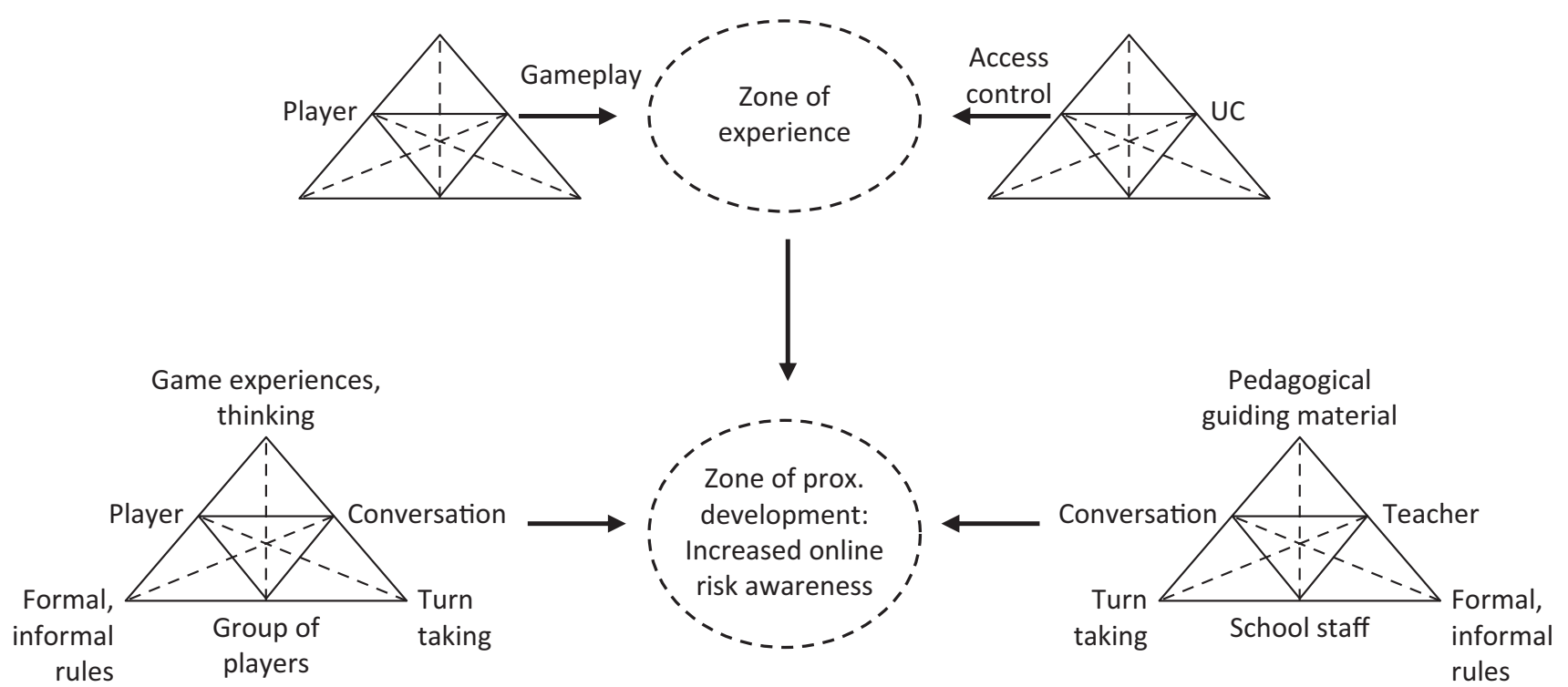

rules

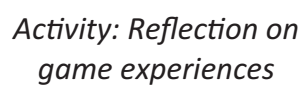

Activity: Aid pupils' reflections on online interactions

Figure 6. The zone of experience is a foundation for the ensuing follow-up conversation in the zone of proximal development, where players and teacher reflect on the gaming experience in relation to online interactions.

one hand are the pupils who reflect on their gaming experience, and on the other hand is the teacher who aids the players/pupils to reflect on the game events and online interactions.

The overall object in both the teacher and player activities is the follow-up conversation. For the players, the mediating tools are their gaming experiences and their reasoning and thinking skills, while the teacher's main mediating tool is the pedagogical guiding material. The players' community is the group of players who have just played the game, and their relationship is mediated by regulations and informal rules and norms. The community for the teacher is other staff members where the rules for being a member of that community is mediated by laws, local regulations and informal norms and agreements. There are clearly numerous kinds of division of labour, especially among staff members, but the one coinciding division of labour between players and teacher is turn taking. As these two activities meet, the common ground and desired outcome, becomes a zone of proximal development where young children can gain raised online risk awareness.

\section{Conclusions}

To combine analogue and digital features into a mixed media game experience certainly presented some challenges. The benefit, in this case, of merging the analogue and the digital to create the game was that it enabled the creation of a socially engaging game that also simulates real world online events, in a way that is not possible if only one or the other had been used. On the one hand, had the game only been digital, the tactile aspect of moving game pieces etc., and the social dynamics of a face to face gameplay situation would have been lost. On the other hand, had the game only been analogue, it would not have had the anchoring in contemporary technology and media usage, such as surprise SMSs, digital photography and the added thrill of AR-technology. The combination of different media allows players to gather around a table in a dynamical social play situation, where the players engage in each other's gameplay for instance, by helping out to move game pieces and reason about choices. The game board also provides a shared, easily accessible overview of the state of the game. Even though the combination of all these aspects - the technologies used, the tangibility of objects, young people's media usage and the social play situation-does provide a fun and engaging play experience, there is no guarantee it will always be the case. After all, we cannot design experiences, we can only design for a good user experience (Hassenzahl \& Tractinsky, 2006).

To conceptualise the design required synchronisation of analogue and digital components, and also the alignment of contradictory but intertwined activities that should meet within the same frame of gameplay. For this we used the activity system model, which allowed both conceptualisation and visualisation of the game concept, and it provided a shared common ground between the developers' widely different fields of competence. It proved a valuable tool for communication within the development team as well as a tool for design, where the relations between the elements within an activity could be analysed. The activity model can aid an analy- 
sis on effects and consequences of changes, for instance, the way a change of game rules affects the relationship between a player and the community of players, which in turn will affect the activity as such. Hence, the model can be utilised to solve inner contradictions within an activity. To solve the issues and contradictions between players' and UCs' differing activities, we used the WOz method during real-time gameplay, which was valuable for identification of interruptions and for the synchronisation of game events. The final product is a mixed media serious game that combines players' and agents' different objectives. It is also a game that draws on the well-known while adding novelty: Board games are familiar and provide tangibility and player engagement, while technology adds a level of something new and exciting. The technology also provides a means for simulating real-world online events, and to control game events and the pacing of the game.

More importantly, however, the zone of experience that a game session establishes creates an opportunity for a situation of insight through the experience of choices and their consequences, of sharing photographs, and to be deceived. We argue this creates the foundation for an activity within the zone of proximal development as described above, in which the gaming experiences are put in relation to real-world online activities under the guidance of an adult. Since the game mimics the target group's everyday media use, where it is not uncommon that children are asked to send photographs to online contacts (Susi et al., 2019), children can potentially increase their thinking skills to better identify and comprehend online risk behaviour.

In sum, the game mimics real-world online events and it is designed for 8-10 year olds, which is a common age when children begin to interact online. The gameplay draws from authentic online sexual grooming offender behaviours, that players are subjected to (without any sexual insinuations or content), thereby providing a zone of experience where players are faced with the negative outcomes of some of their decisions. The follow up conversation brings the experiences into the zone of proximal development, where players can discuss and reflect on game events. All these intricate activities and processes are clarified through the activity system model that contextualises the individual players within a wider sociocultural context. Furthermore, the activity system model can be a useful tool for design communication, and it can aid the analysis on the effects and consequences of changes in a design process.

Hidden in the Park can very well be played as just a fun and entertaining stand-alone game, as our evaluations clearly showed, but as a means to raise online risk awareness the follow-up discussion is fundamental for the game to reach its full potential. The game is distributed free of charge to all elementary schools in Sweden since 2019.

To return to Aarseth's (2001) thoughts on digital games as a unique media form, we agree but we also believe that analogue components combined with digital ones can add a further dimension to the gaming experience. The development of mixed media provides good opportunities to expand the experience of games in a way that perhaps no media on its own can do. We firmly believe the game discussed here is a tool that offers "an insight into the ways that games work to produce social change" (Torner et al., 2014). The game establishes a zone of experience that has a great potential to form young people's internet behaviour and online risk awareness. We believe mixed media has the potential to provide compelling platforms and various forms of games and thereby tease out good conditions for affecting behaviours for the benefit of social sustainability. This is a good reason for game study research to attend to the advantages of mixed media, because surely it is an exciting development that awaits around the corner.

\section{Acknowledgments}

The project was carried in two parts, as a joint venture between the University of Skövde, the non-profit organisation The Change Attitude Foundation, and the game company IUS Innovation. The first project was financed by the Sten A Olsson Foundation for Research and Culture. The second project was financed by the World Childhood Foundation and the University of Skövde. The research team includes the following people at the University of Skövde: Prof. Per Backlund, Assoc. Prof. Ulf Wilhelmsson, PhD student Marcus Toftedahl, Research Engineer Mikael Lebram, Dr. Niklas Torstensson, and Dr. Tarja Susi. We would also like to thank our anonymous reviewers for valuable and constructive comments.

\section{Conflict of Interests}

The authors declare no conflict of interests.

\section{References}

Aarseth, E. (2001). Computer game studies, year one. Game Studies, 1(1), 1-15.

Antle, A. N. (2013). Exploring how children use their hands to think: An embodied interaction analysis. Behavior \& Information Technology, 32(9), 938-954.

Caillois, R. (2001). Man, play and games (Meyer Barash, Trans.). Champaign, IL: University of Illinois Press. (Original work published 1958)

Carvalho, M. B., Bellotti, F., Berta, R., De Gloria, A., Sedano, C. I., Hauge, J. B., . . Rauterberg, M. (2015). An activity theory-based model for serious games analysis and conceptual design. Computers \& Education, 87, 166-181.

Dow, S., Maclntyre, B., Lee, J., Oezbek, C., Bolter, J. D., \& Gandy, M. (2005). Wizard of Oz support throughout an iterative design process. IEEE Pervasive Computing, 4(4), 18-26.

Engeström, Y. (1987). Learning by expanding: An activity- 
theoretical approach to developmental research. Helsinki: Orienta-Konsultit Oy.

Goffman, E. (1986). Frame analysis: An essay on the organization of experience. Boston, MA: North Eastern University Press.

Hassenzahl, M., \& Tractinsky, N. (2006). User experience: A research agenda. Behaviour \& Information Technology, 25(2), 91-97.

Höysniemi, J., Hämäläinen, P., \& Turkki, L. (2004). Wizard of Oz prototyping of computer vision based action games for children. In A. Druin, J. P. Hourcade, \& S. Kollet (Eds.), Proceedings of the 2004 Conference on Interaction Design and Children: Building a Community (pp. 27-34). New York, NY: ACM.

Huizinga, J. (1955). Homo ludens: A study of the playelement in culture. Boston, MA: Beacon Press.

Kankainen, V., Arjoranta, J., \& Nummenmaa, T. (2017). Games as blends: Understanding hybrid games. Journal of Virtual Reality and Broadcasting, 14(4).

Kozulin, A. (1996). The concept of activity in Soviet psychology: Vygotsky, his disciples and critics. In H. Danies (Ed.), An introduction to Vygotsky (pp. 99-122). London: Routledge.

Salen, K., \& Zimmerman, E. (2003). Rules of play: Game design fundamentals. Cambridge, MA: The MIT Press.

Susi, T. (2006). The puzzle of social activity: The significance of tools in cognition and cooperation. Linköping: Department of Computer and Information Science, University of Linköping.

Susi, T., Johannesson, M., \& Backlund, P. (2007). Serious games: An overview. (Technical Report HS-IKI-TR-07001). Skövde: University of Skövde.

Susi, T., \& Torstensson, N. (2019). "Who's texting?" Playful game experiences for learning to cope with online risks. In X. Fang (Ed.), Proceedings of the Internation- al Conference on Human-Computer Interaction (pp. 427-441). Cham: Springer.

Susi, T., Torstensson, N., \& Wilhelmsson, U. (2019). “Can you send me a photo?": A game-based approach for increasing young children's risk awareness to prevent online sexual grooming. In A. Nakamura (Ed.), DiGRA 19: Proceedings of the DiGRA 2019 International Conference: Game, Play and the Emerging Ludo-Mix, (pp. 1-16). Kyoto: Digital Games Research Association.

The Change Attitude Foundation. (2019). Hidden in the Park [Video Game]. Skövde: IUS Innovation.

The Swedish Media Council. (2017a). Småungar \& medier [Small kids \& media]. Stockholm: The Swedish Media Council.

The Swedish Media Council. (2017b). Ungar \& medier [Kids \& media]. Stockholm: The Swedish Media Council.

Torner, E., Trammel, A., \& Leigh Waldron, E. (2014). Reinventing analog game studies. Analog Game Studies, 1(1).

Torstensson, N., Susi, T., Wilhelmsson, U., \& Lebram, M. (2020). Wizard of $\mathrm{Oz}$ and the design of a multi-player mixed reality game. In X. Fang (Ed.), Proceedings of the International Conference on Human-Computer Interaction 2020. Cham: Springer.

United Nations. (2015). Transforming our world: The 2030 agenda for sustainable development. New York, NY: United Nations. Retrieved from https://sustainabledevelopment.un.org/post2015/ transformingourworld

Vygotsky, L. S. (1978). Mind in society: The development of higher psychological processes. Cambridge, MA: Harvard University Press.

Wertsch, J. V. (1981). The concept of activity in Soviet psychology. North Castle, NY: M. E. Sharpe.

\section{About the Authors}

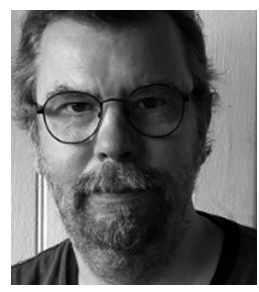

Ulf Wilhelmsson is Associate Professor in media, aesthetics and narration at the University of Skövde, Sweden. He has worked in serious games projects within cultural heritage, raised online risk awareness for young children, and inclusive game design. He is a member of the research group Media, Technology and Culture at the School of Informatics, University of Skövde, Sweden.

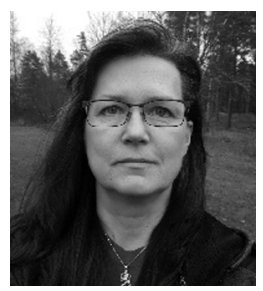

Tarja Susi is a Senior Lecturer in cognitive science at University of Skövde, Sweden. She has worked in serious games projects within cultural heritage, raised online risk awareness for young children, and eSports. She is a member of the research group Media, Technology and Culture at the School of Informatics, University of Skövde, Sweden. 


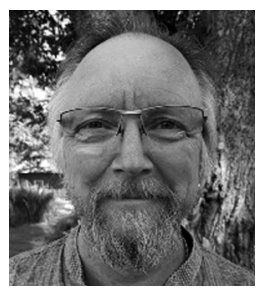

Niklas Torstensson is a Senior Lecturer in Cognitive Science at University of Skövde, Sweden. He has worked in serious games projects within cultural heritage, and raised online risk awareness for young children and esports. He is a member of the research group Media, Technology and Culture at the School of Informatics, University of Skövde, Sweden. 